\title{
Piezoceramic Transducers Selection Method for High Power Ultrasonic Devices
}

\author{
P. KLUK* AND A. MILEWSKI \\ Tele and Radio Research Institute, Ratuszowa 11, 03-450 Warszawa, Poland \\ (Received May 12, 2014)
}

A universal method for selecting piezoceramic transducers used in high power ultrasonic devices such as ultrasonic welding and cutting systems has been presented. The key element of such systems is a high power ultrasonic sandwich transducer consisting of a stack of piezoceramic transducers mounted between two masses. Because of relatively large dispersion of piezoceramic transducer parameters between production lots, they must be selected in order to obtain a high quality and long-life sandwich transducer. Presented selection method consists of three stages. First, the parametric identification of the Butterworth-Van Dyke equivalent circuit model is performed based on piezoceramic transducer electrical impedance measurements. Next, the electrical impedance frequency samples are estimated using the identified Butterworth-Van Dyke model. Finally, the $k$-means clustering algorithm is used to select the best fitted piezoceramic transducer sets.

DOI: $10.12693 /$ APhysPolA.127.719

PACS: 43.35.+d, 43.35.-c, 43.35.Zc, 43.38.Fx, 43.38.+n, 43.38.-p

\section{Introduction}

Piezoceramic transducers (rings) are the main building blocks of high power ultrasonic sandwich transducers [1]. A batch sample of piezoceramic transducers has been presented in Fig. 1. Usually, the sandwich transducer is composed of a stack of two, four or six piezoceramic transducers mounted between two cylindrical metal resonators (an emitter and a reflector). Examples of sandwich transducer physical models have been presented in Fig. 2. This kind of ultrasonic transducer is one of the most vital parts of high power ultrasonic devices, such as contemporary ultrasonic welding and cutting systems. These transducers deserve to be called "heavy duty" transducers taking into account their following working conditions: real power up to $2-6 \mathrm{~kW}$, RMS voltage up to $3.2 \mathrm{kV}$, RMS current up to $3 \mathrm{~A}$, in a frequency range of $18 \mathrm{kHz}$ to $80 \mathrm{kHz}$. To manufacture a high quality and long-life sandwich transducer we need to iteratively optimize its geometry and select adequate piezoceramic material type in the design process [1-3]. But, even if we do it perfectly, there is a one important problem left, i.e. relatively large dispersion of parameters in piezoceramic transducers production lots (batches). To illustrate this problem, impedance characteristics for a batch sample of 19 piezoceramic transducers (piezoceramic material type: NCE40, dimensions: $\left.20 \times 10 \times 1 \mathrm{~mm}^{3}\right)$ have been presented in Fig. 3 . The impedance characteristics have been measured using measurement system presented in [4]. It is not difficult to note that a random selection of transducers from this batch sample is not a good idea. For example, the resultant sandwich transducer quality factor should be as high as possible which can be achieved only if all the piezoceramic transducers in the stack have similar resonance frequencies and quality factors.

\footnotetext{
${ }^{*}$ corresponding author; e-mail: piotr.kluk@itr.org.pl
}

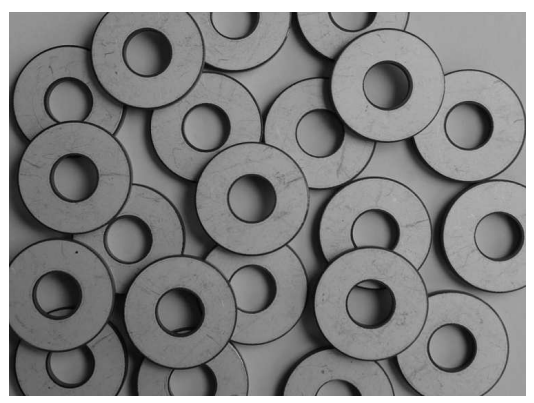

Fig. 1. Piezoceramic transducers batch sample.

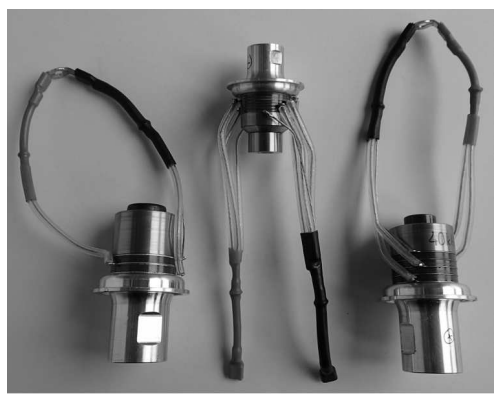

Fig. 2. Sandwich transducer physical models.

\section{Electrical impedance compatibility as the selection criterion}

For the same geometry (shape and dimensions) of piezoceramic transducers and infinite homogeneity of given piezoceramic material the impedance characteristics presented in Fig. 3 should be identical (overlapping each other), which corresponds to ideal compatibility of transducers parameters. For example, let us try to select the best-matched and worst-matched pairs in the batch sample. There is a pair whose impedance curves are overlapping each other, i.e. they are best-matched, which has been presented in Fig. 4. The impedance curves of the worst-matched transducer pair have been presented in Fig. 5. 


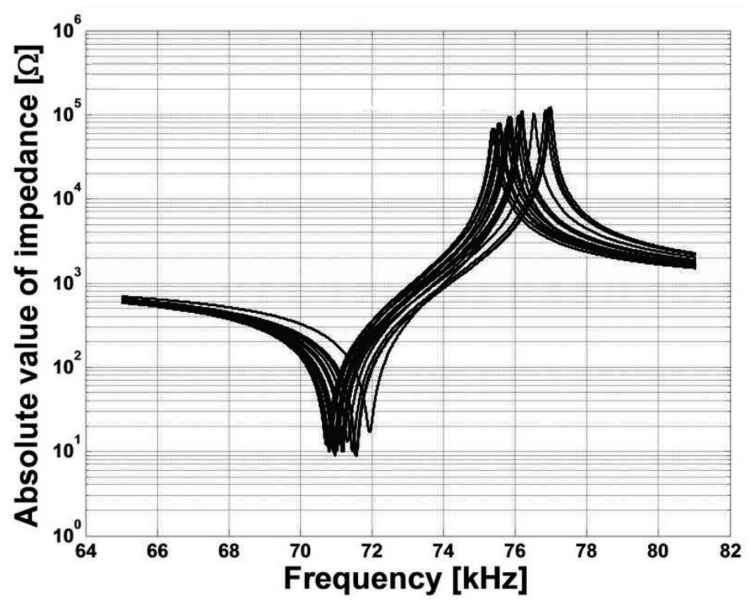

Fig. 3. Impedance curves of 19 piezoceramic transducers batch sample.

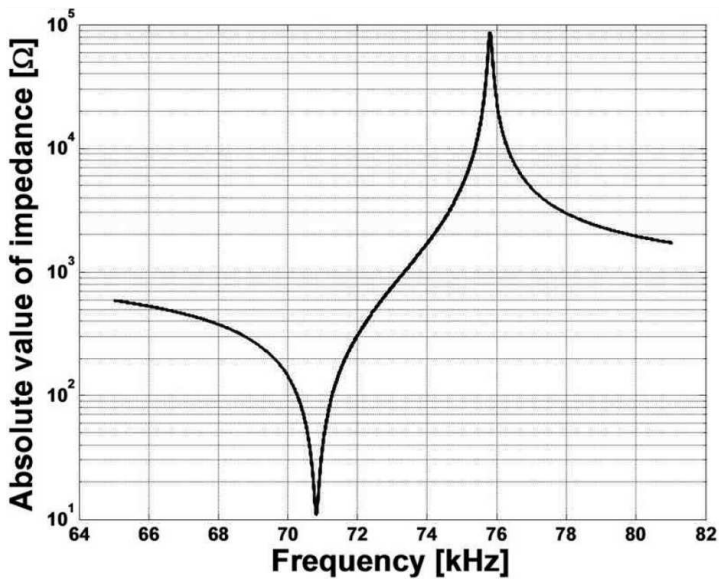

Fig. 4. Overlapping impedance curves indicate bestmatched transducers.

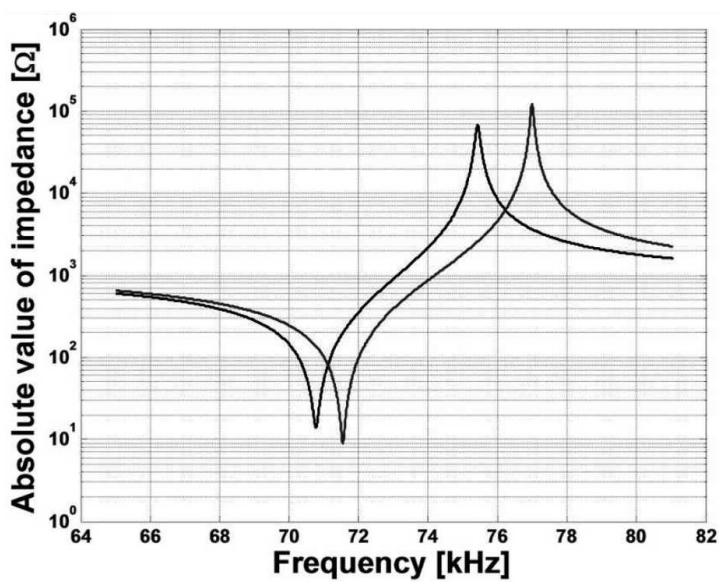

Fig. 5. The most distant impedance curves indicate worst-matched transducers.

\subsection{Using series and parallel resonance parameters as} the selection criteria

For the best-matched pair the following relations are true:

$$
\begin{aligned}
& \left(f_{\mathrm{s}, 1},\left|Z_{\mathrm{s}, 1}\right|\right) \cong\left(f_{\mathrm{s}, 2},\left|Z_{\mathrm{s}, 2}\right|\right), \\
& \left(f_{\mathrm{p}, 1},\left|Z_{\mathrm{p}, 1}\right|\right) \cong\left(f_{\mathrm{p}, 2},\left|Z_{\mathrm{p}, 2}\right|\right),
\end{aligned}
$$

where the points $\left(f_{\mathrm{s}},\left|Z_{\mathrm{s}}\right|\right)$ and $\left(f_{\mathrm{p}},\left|Z_{\mathrm{p}}\right|\right)$ denote the series and parallel resonances, respectively. So, one can try to match the series and parallel resonance points only, as it has been proposed in [5], but generally, it leads to not-easy-to-deal multi-objective optimization and Pareto-optimal points. Let us take a look to the worst-matched pair to explain such a situation. For the worst-matched pair the following relations are true:

$$
\left(f_{\mathrm{s}, 1} \neq f_{\mathrm{s}, 2}\right) \wedge\left(\left|Z_{\mathrm{s}, 1}\right| \neq\left|Z_{\mathrm{s}, 2}\right|\right),
$$

$$
\left(f_{\mathrm{p}, 1} \neq f_{\mathrm{p}, 2}\right) \wedge\left(\left|Z_{\mathrm{p}, 1}\right| \neq\left|Z_{\mathrm{p}, 2}\right|\right) .
$$

That is, for the worst-matched pair their corresponding resonance frequencies are different as well as the absolute values of impedances at those frequencies are different. So, if we try to improve impedance compatibility of a randomly selected pair we can find pairs with better resonance-frequency compatibility but worse absolutevalue-of-impedance compatibility, and vice versa, which leads us to not easy task of looking for Pareto-optimal points.

\subsection{Using Euclidean distance to measure impedance compatibility}

In practice, we have a series of $N$ impedance samples, as has been presented in Fig. 6, measured over a specified frequency range

$$
\left|Z\left(f_{n}\right)\right|, \text { for } \mathrm{n}=1 \ldots \mathrm{N} \text {. }
$$

If we treat the discrete impedance characteristic as a point $\boldsymbol{x}$ of $N$-dimensional Euclidean space $R^{N}$, i.e.

$$
\boldsymbol{x}=\left(Z\left|\left(f_{1}\right)\right|,\left|Z\left(f_{2}\right)\right|, \ldots,\left|Z\left(f_{N}\right)\right|\right),
$$

then we can use the following metric (distance function) to measure distance between two points $\boldsymbol{x}$ and $\boldsymbol{y}$ :

$$
d_{\mathrm{E}}(\boldsymbol{x}, \boldsymbol{y})=|\boldsymbol{x}-\boldsymbol{y}|=\sqrt{\sum_{n=1}^{N}\left(x_{n}-y_{n}\right)^{2}} .
$$

The Euclidean metric (7) is a good objective function for selecting well-matched transducer pairs but is not directly applicable for matching more numerous sets of transducers.

\subsection{Using k-means clustering algorithm to measure impedance compatibility}

Given a set of $P$ points $\left\{x_{1}, x_{1}, \ldots, x_{P}\right\}$ of $N$ dimensional Euclidean space $R^{N}$, find $K(K \leq P)$ subsets $S=\left\{S_{1}, S_{1}, \ldots, S_{K}\right\}$ in the following way [6]:

$$
\underset{\mathrm{S}}{\arg \min }\left\{\sum_{k=1}^{K} \sum_{\boldsymbol{x}_{m} \in S_{k}}\left\|\boldsymbol{x}_{m}-\boldsymbol{\mu}_{k}\right\|^{2}\right\},
$$




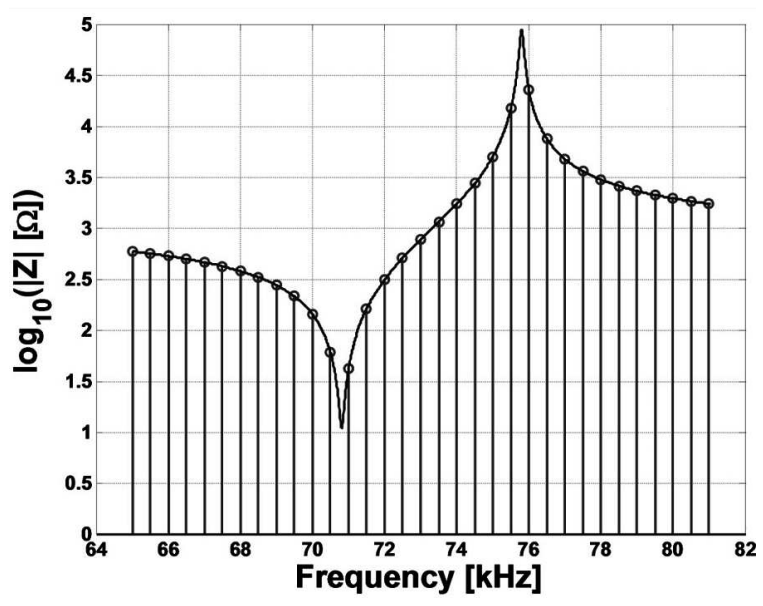

Fig. 6. Series of $N=33$ impedance samples, $f_{1}=$ $65 \mathrm{kHz}, f_{33}=81 \mathrm{kHz}$.

$$
\boldsymbol{\mu}_{k}=\frac{1}{M} \sum_{m=1}^{M} \boldsymbol{x}_{m}
$$

where the $\mu_{k}$ vector is co called "mean" or "centroid" of the $k$-th cluster. We can use other clustering algorithm, such as SOM neural network, fuzzy $c$-means or traditional hierarchical clustering algorithms, as well, but the $k$-means algorithm is widely used and gives good outcomes [6].

The meaning (interpretation) of $k$-means algorithm parameters in our problem is the following: $P-$ number of piezoceramic transducers for selection, $N$ - number of impedance curve samples, $K$ - number of best-matched transducer groups (clusters), $M$ - number of transducers per cluster (known a priori), $\mu_{k}$ - expected values of impedance curve samples for $k$-th cluster (impedance curve pattern or reference impedance curve).

Therefore, we can use the standard $k$-means clustering algorithm directly with one additional constraint, i.e. a fixed number of members per cluster.

Minimizing the functional (8) gives the best partitioning of a given transducers batch in the least-squares sense. It is a good solution if we want to effectively utilize the entire transducers batch. But sometimes, e.g. for examination purposes, we want to select the best-matched groups of transducers. For example, let us partition the set of $19(P=19)$ piezoceramic transducers into 4element subsets $(M=4)$, starting from the best-matched set and ending with the worst-matched set. To do so, we can use the $k$-means algorithm in the following way:

- For each 4-combination without repetition of the piezoceramic transducer set compute the "distance" $d_{j}$ according to the following formulae:

$$
\begin{aligned}
& d_{j}=\sum_{m=1}^{4}\left\|\boldsymbol{x}_{m}-\boldsymbol{\mu}_{j}\right\|^{2} \boldsymbol{\mu}_{j}= \\
& \frac{1}{4} \sum_{m=1}^{4} \boldsymbol{x}_{m}, \text { for } j=1, \ldots, J .
\end{aligned}
$$

- Sort the distances $d_{j}$ in ascending order;

- The smallest distance corresponds to the bestmatched group;

- The greatest distance corresponds to the worstmatched group;

- Select $K$ transducer groups starting from the best-matched one and rejecting groups including transducers already selected

$$
K=\operatorname{floor}(\mathrm{P} / \mathrm{M})=4 \text {. }
$$

The main problem is the numerical complexity because of the number $J$ of all possible $M$-combinations, which can be evaluated in the following way:

$$
J=\left(\begin{array}{c}
P \\
M
\end{array}\right)=\left(\begin{array}{c}
19 \\
4
\end{array}\right)=93024
$$

For example, impedance curves of the best-matched and worst-matched quadruplets of the batch sample have been presented in Fig. 7, along with their normalized mismatch measure values.

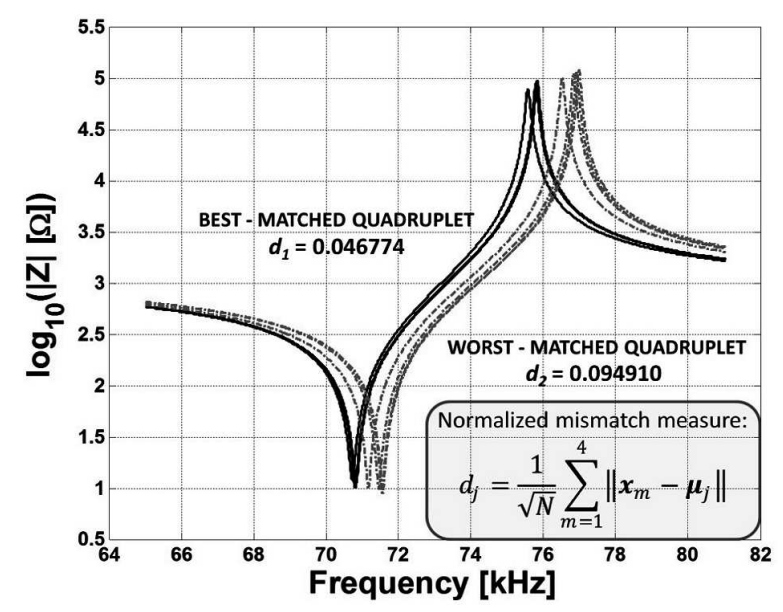

Fig. 7. Best-matched and worst-matched quadruplets of the batch sample.

\section{Using the BVD model for measurement data preprocessing (smoothing and resampling)}

It should be noted that presented method assumes explicitly equinumerous impedance samples sets taken at the same frequency points, according to (5). In some circumstances, the raw measurement data from measurement system cannot meet these requirements. In such a case, we should approximate the raw measurement data using, for example, the well known ButterworthVan Dyke equivalent circuit (BVD model) [1-3]. There is no need to use more sophisticated, i.e. modified or generalized BVD [7], models. The BVD model leads to the following formula for impedance: 


$$
Z(f)=\frac{R+j\left(2 \pi f L-\frac{1}{2 \pi f C}\right)}{2 \pi f C_{0}\left(\frac{1}{2 \pi f C}+\frac{1}{2 \pi f C_{0}}-2 \pi f L+j R\right)} .
$$

An exemplary impedance curve approximation has been presented in Fig. 8. The BVD model parametric identification has been conducted using the LevenbergMarquardt optimization algorithm [8]. The starting point has been evaluated using formulae presented in [9]. As we can see, an additional parasite resonance (near the right side of the series resonance), generated as a consequence of non-neglible test-fixture clamping force, has been efficiently smoothed.

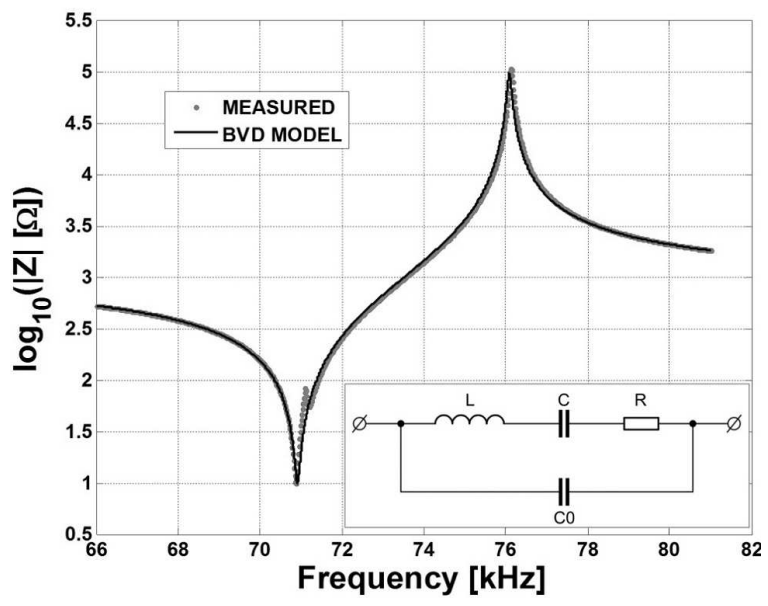

Fig. 8. Impedance curve approximation using BVD model.

\section{Conclusions}

The presented method allows the automatic and fast selection of piezoceramic transducer sets designed to operate in a stack, resulting in an improvement of "Sandwich" transducer parameters. This made it possible to improve the performance and uptime of high-power ultrasonic devices, e.g. ultrasonic welding and cutting systems, in industrial applications.

\section{Acknowledgments}

We gratefully acknowledge the financial support of the National Centre for Research and Development, Poland, under grant number PBS2/B9/19/2013.

\section{References}

[1] M.Đ. Radmanović, D.D. Mančić, Designing and Modelling of the Power Ultrasonic Transducers, MPI, Switzerland 2004.

[2] M. Prokic, Piezoelectric Transducers Modeling and Characterization, MPI, Switzerland 2004.

[3] A. Arnau, Piezoelectric Transducers and Applications, Springer-Verlag, Berlin 2008.

[4] P. Kluk, A. Milewski, W. Kardyś, P. Kogut, P. Michalski, Acta Phys. Pol. A 124, 468 (2013).

[5] P.A. Senyutkin, E.F. Chineikina, Russ. J. Nondestruct. Test. 39, 115 (2003).

[6] S.A. Mingoti, J.O. Lima, Europ. J. Operat. Res. 174, 1742 (2006).

[7] H. Jin, S.R. Dong, J.K. Luo, W.I. Milne, Electron. Lett. 47, 424 (2011).

[8] R. Fletcher, Practical Methods of Optimization, Wiley, Chichester 2000.

[9] A. Milewski, P. Kluk, P. Kogut, J. FlorkowskaTrąbińska, Elektronika 52, 194 (2011) (in Polish). 